\title{
Viral Pneumonia on Computed Tomography Thorax Revisited in Covid-19 Era
}

\author{
Rajul Rastogi, ${ }^{1, *}$ Neha1, Vijai Pratap ${ }^{1}$, Sujeet Kumar Jain ${ }^{2}$ \\ ${ }^{1}$ Department of Radiodiagnosis, Teerthanker Mahaveer Medical College \& Research Center, Moradabad, Uttar Pradesh, India. \\ ${ }^{2}$ Department of Radiodiagnosis, School of Medical Sciences \& Research and Sharda Hospital, Greater Noida, Uttar Pradesh, In- \\ dia.
}

How to cite this paper: Rajul Rastogi, Neha, Vijai Pratap, Sujeet Kumar Jain. (2020) Viral Pneumonia on Computed Tomography Thorax Revisited in Covid-19 Era. International Journal of Clinical and Experimental Medicine Research, 4(3), 73-78.

DOI: 10.26855/ijcemr.2020.07.008

Received: June 5, 2020

Accepted: June 30, 2020

Published: July 10, 2020

*Corresponding author: Rajul Rastogi, Associate Professor, Department of Radiodiagnosis, Teerthanker Mahaveer Medical College \& Research Center, Moradabad, Uttar Pradesh, India.

Email: eesharastogi@gmail.com

\begin{abstract}
The novel severe acute respiratory syndrome coronavirus, i.e., SARS-CoV-2 is responsible for ongoing Covid-19 pandemic. Though the morbidity and mortality related to SARS-CoV-2 is reported to be far less than previous pandemics caused by influenza, SARS-CoV and Middle East respiratory syndrome virus in recent medical literature yet its highly contagious nature is of great concern. In addition, the low sensitivity of highly specific real-time polymerase chain reaction to detect viral nucleotides in the samples from oropharynx, nasopharynx \& tracheobronchial tree coupled with viral transmission ability of asymptomatic individuals has made it imperative for computed tomography of thorax to help in diagnosis of Covid-19 not only for early institution of management in serious cases but also to help quarantine the asymptomatic carriers. Hence, this article revisits lung findings on computed tomography in common viral pneumonias and attempts to bring out key differentiating lung findings of this new pandemic disease.
\end{abstract}

\section{Keywords}

Viral pneumonia, computed tomography thorax, Covid-19

\section{Introduction}

Covid-19 is a viral illness caused by severe acute respiratory syndrome coronavirus type-2 (SARS-Cov-2) [1]. Several reports in recent medical literature reveal significant role of non-contrast computed tomography imaging of thorax (CT-Th) in diagnosis and management of Covid-19 pneumonia [2].

The predominant findings of Covid-19 pneumonia on CT-Th are diffuse, bilateral \& peripheral pattern of ground-glass opacification (GGO) or haziness in early stages progressing to consolidation in later stages [3-6]. Significant number of these patients also show vascular thickening of unknown pathogenesis in the areas of GGO along with uncommon air-bronchogram in areas of consolidation [7]. Minority of patients may however present with unilateral and focal disease especially in asymptomatic patients which usually progresses to classical pattern within 1-2 weeks of symptom-onset [3, 8].

However, the varied CT-Th manifestations poses a diagnostic challenge in the absence of positive real-time polymerase chain reaction (RT-PCR) as these findings have a significant overlap with other viral pneumonias [2, 9].

\section{Discussion}

Respiratory tracts infections are usually viral in etiology and their imaging findings have a significant overlap with nonviral bacterial infections as well as with many inflammatory conditions [9]. Though imaging patterns are 
not pathognomonic of any viral etiology, yet they are characteristic of common viral families in immunocompetent and immunocompromised hosts of pediatric and adult age-group [9]. This pattern recognition may help in early institution of antiviral therapy besides avoiding unnecessary antibiotic therapy especially prior to establishing an etiological laboratory diagnosis.

The common viral infectionsin immunocompetent adults in the decreasing order of frequency are influenza virus, human parainfluenza virus (HPIV), adenovirus \& respiratory syncytial virus (RSV) while they include RSV, HPIV, influenza virus \& human metapneumovirus (HMPV) in the same order in pediatric age-group [9]. On the other hand, the common viral infections in immunocompromised adults are influenza virus, RSV, adenovirus \& rhinovirus in the decreasing order of frequency while in pediatric age-group they include adenovirus, cytomegalovirus (CMV), Epstein-Barr virus (EBV) \& HSVin the same order [9].

Key differentiating points that should be noted to determine etiology of various viral pneumonia on CT-Th should include:

- Distribution of GGO (seen in early phase) \& consolidation (seen in intermediate \& later stages) and

- Presence or absence of nodules (usually centrilobular-CLB).

Majority of viral pneumonia shows bilateral, multifocal pattern (patchy with segmental or lobar) of both GGO (Image $1 \& 2$ ) and consolidation (Image 3) except in CMV \& EBV where the pattern is usually diffuse although pneumonia is rare in EBV [9]. Small areas of consolidation may become confluent and coalescent to form larger areas with air-bronchogram (Image 4). Cavitation is however uncommon in these areas unlike bacterial pneumonia.

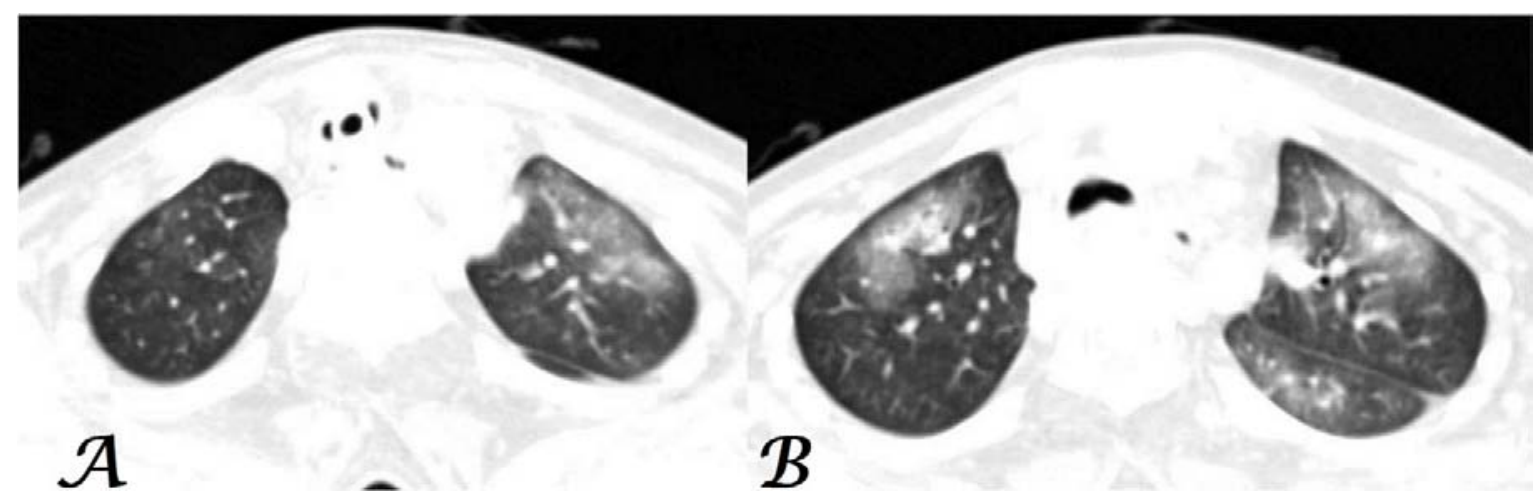

Image 1a-b. Transaxial CT images in lung window settings show dominant \& peripheral, unifocal GGO in apical segment of LUL (a) and multifocal, multisegmental \& peripheral GGO involving bilateral upper lobes (b) seen in viral pneumonia.

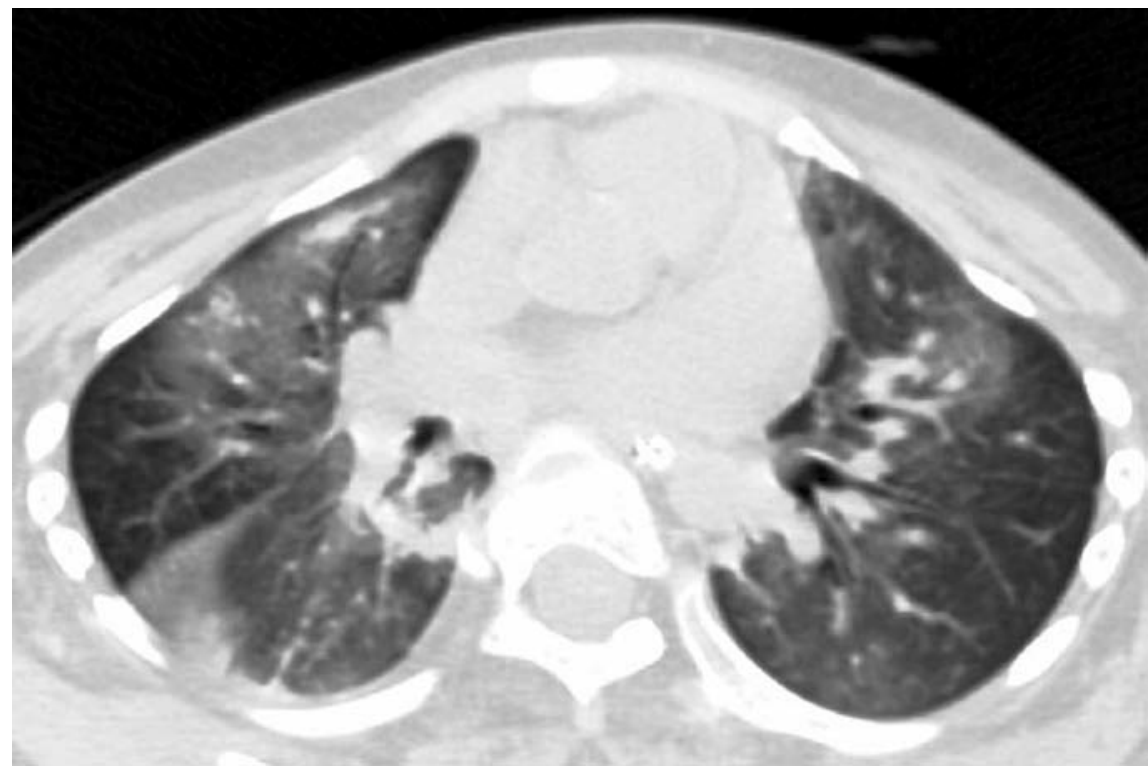

Image 2. Transaxial CT image in lung window settings shows bilateral, diffuse, peripheral GGO in a case of viral pneumonia. 


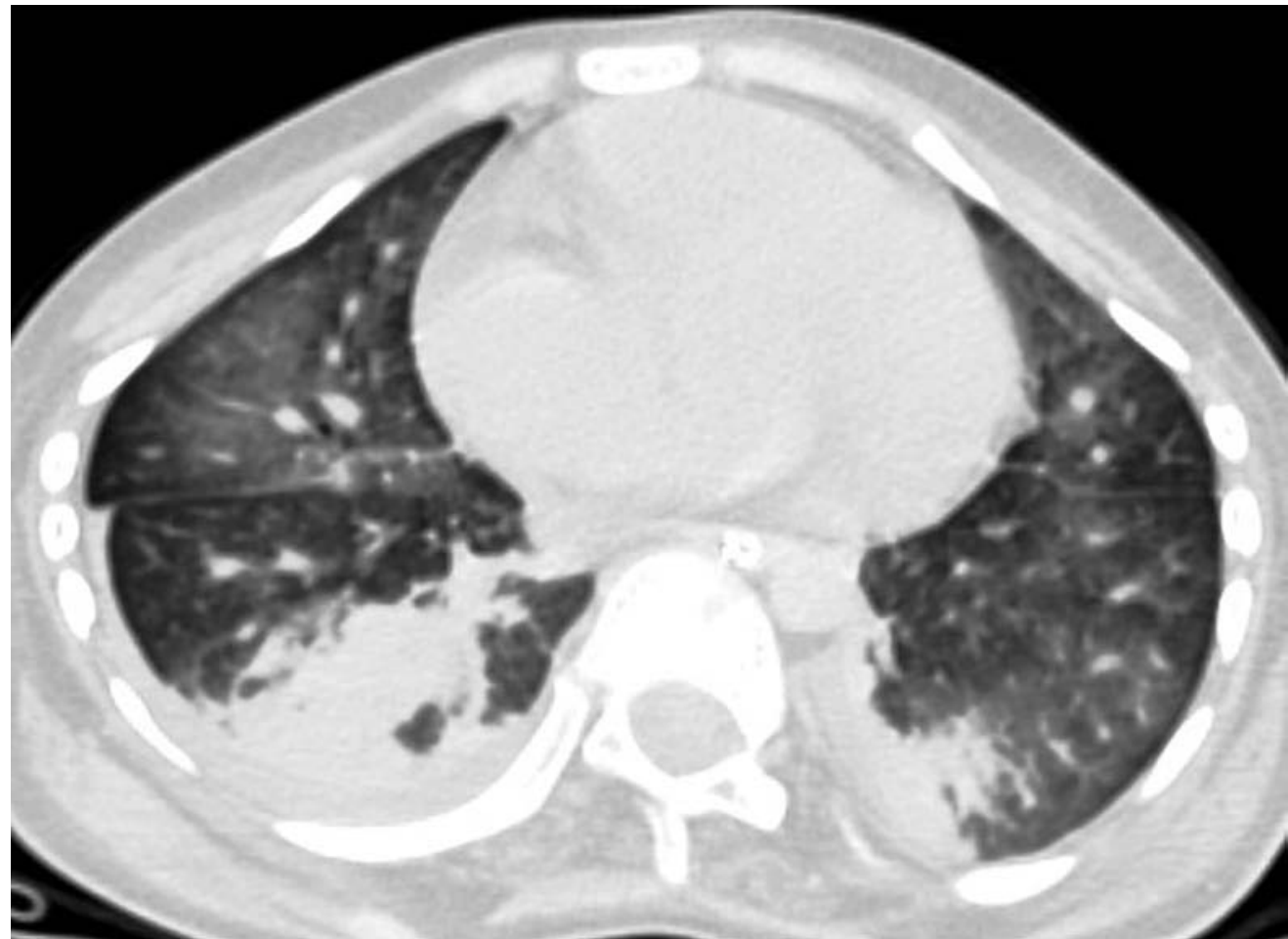

Image 3. Transaxial CT image in lung window settings shows bilateral, diffuse \& peripheral GGO with consolidation in bilateral lower lobe segments and minimal pleural effusion on right side in a case of viral pneumonia.

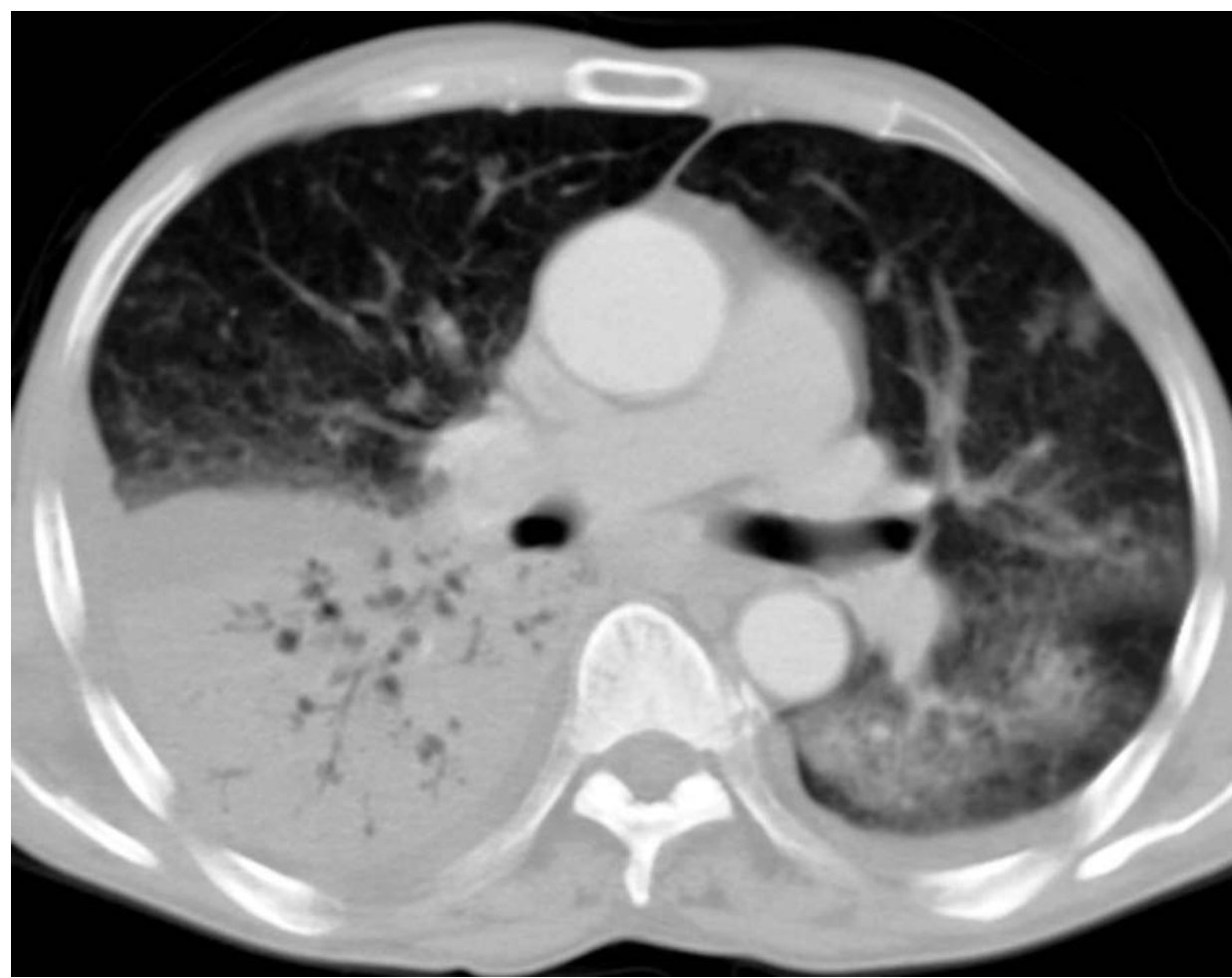

Image 4. Transaxial CT image in lung window settings shows multifocal GGO with consolidation in bilateral lower lobe segments; more marked on the right side with air bronchogram and minimal pleural effusion in a case of viral pneumonia.

CLB nodules (Image 5) are most noted with RSV \& HMPV followed by influenza, HPIV, adenovirus \& HSV but are rare with coronavirus (CoV), rhinovirus \& EBV [9]. However, calcified nodules in CLB distribution is quite characteristic sequelae of varicella-zoster pneumonia. 


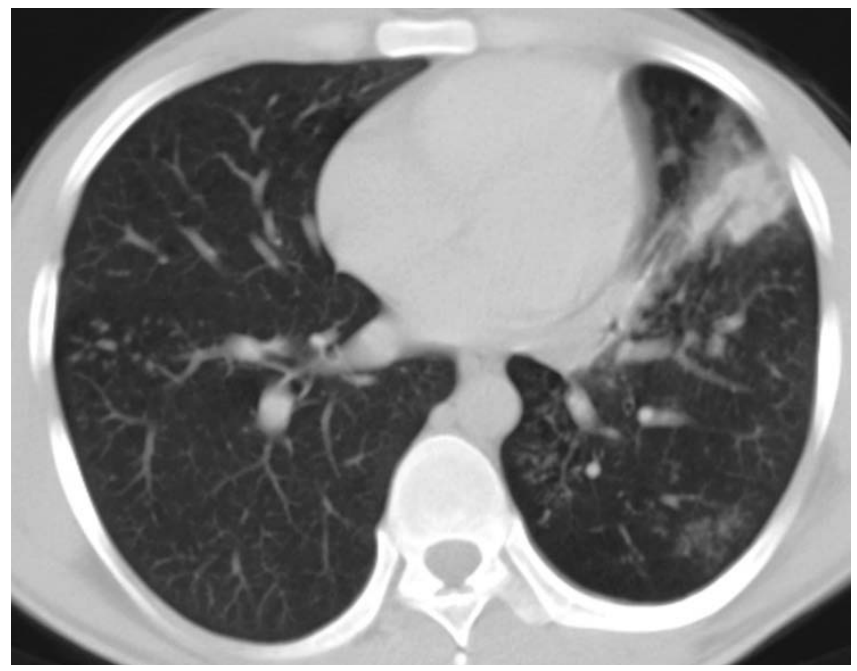

Image 5. Transaxial CT image in lung window settings shows focal GGO in left lower lobe with focal segmental consolidation in left upper lobe and multifocal centrilobular nodules in basal segments of bilateral lower lobes in a case of viral pneumonia.

Though not key features yet presence of bronchial wall thickening, commonly noted with influenza virus, HPIV, RSV \& HPMV pneumonia and pleural effusion characteristically seen with adenovirus \& RSV can also be considered as supportive and ancillary findings aiding in better characterisation of overlapping viral pneumonia patterns [9].

Coronavirus family which includes SARS-CoV, Middle East Respiratory Syndrome (MERS) \& SARS-CoV-2 are known to cause pneumonia characterised by predominant peripheral distribution of GGO \& consolidation progressing to reticulations and fibrosis (Image 6) in later or recovery stages on CT-Th [10]. Unifocal rather than multifocal or bilateral pattern on CT-Th at the time of presentation is characteristic of SARS-CoV progressing to become multifocal \& bilateral in later stages [9]. Besides the above, MERS is commonly associated with lymphopenia and pleural effusion or pneumothorax on CT-Th in severe cases [11]. Bilateral GGO and consolidation in contrast to Covid-19 was an early feature of SARS-CoV while its subpleural \& basilar distribution is characteristic of MERS pneumonia [9, 10].

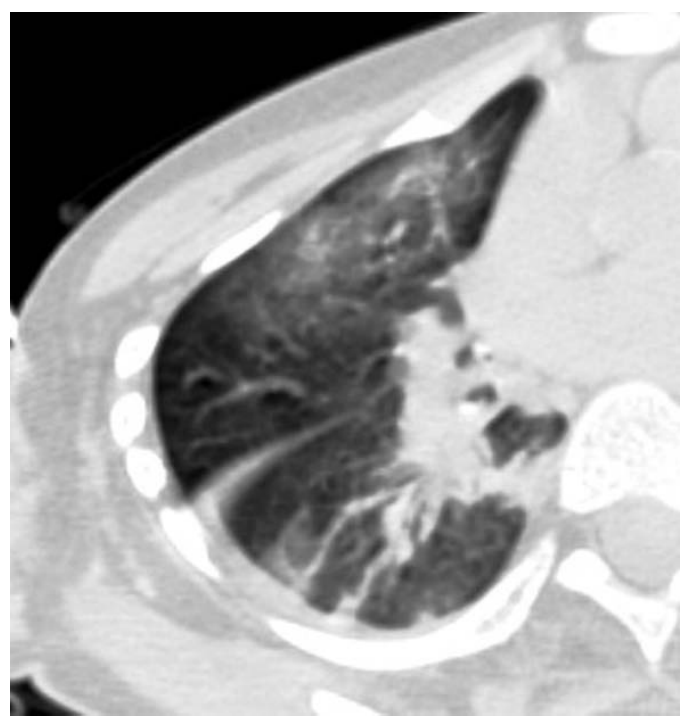

Image 6. Transaxial CT image in lung window settings shows multifocal GGO in right upper lobe along with reticulations/fibrosis in right lower lobe and minimal pleural effusion on right side in a case of viral pneumonia.

Though like non-Covid viral pneumonia, Covid-19 pneumonia is also characterised by diffuse, bilateral and peripheral GGO progressing to consolidation but vascular thickening within GGO (Image 7) \& reverse halo sign (Image 8) had predominance in Covid-19 disease [7]. In contrast, a combination of central \& peripheral distribution of GGO/consolidation and pleural effusion was commoner with non-Covid disease [7]. 


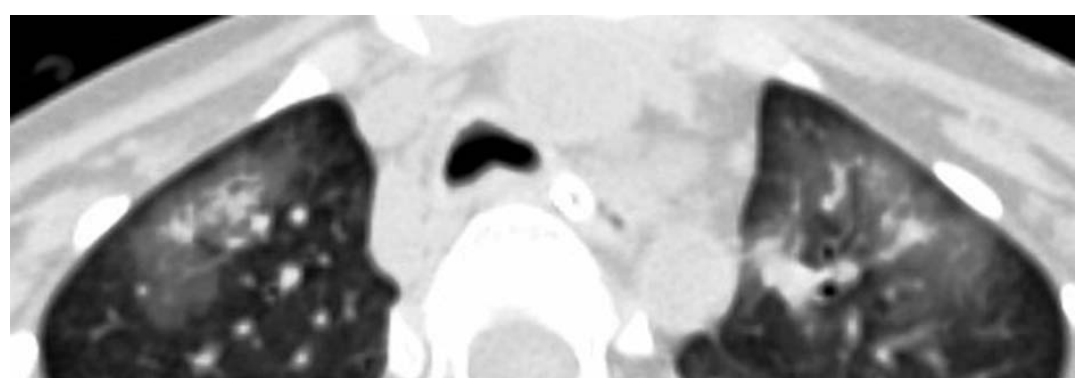

Image 7. Transaxial CT image in lung window settings shows vascular thickening within the peripheral GGO in bilateral upper lobes in a case of viral pneumonia.

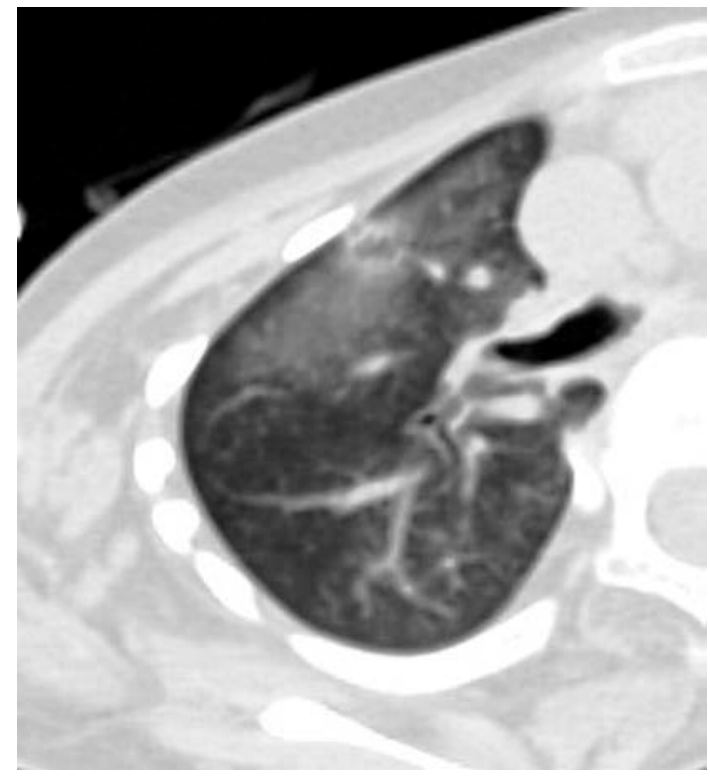

Image 8. Transaxial CT image in lung window settings shows reverse-halo sign in right upper lobe segment in subpleural location along with GGO in a case of viral pneumonia.

Though in initial studies CT-Th appeared to have a high sensitivity \& low specificity for detecting Covid-19 pneumonia but a recent study revealed its high specificity \& moderate sensitivity [7, 8, 12]. Hence, CT-Th can be considered as an imaging tool not only in symptomatic Covid-19 with grave disease to know the extent \& progression of disease but also as a screening tool in asymptomatic patients with false-negative RT-PCR in early phase especially those with travel history to hotspot of Covid-19 disease and contact with infected individual [2].

In early stages of Covid-19 infection in China, CT-Th was included in the diagnostic criteria probably to ensure early treatment by tiding over heavy influx of patients with respiratory symptoms to hospital in short time coupled with inherent delays in laboratory diagnosis [2]. Easy availability of non-contrast CT-Th and artificial intelligence modules might have also influenced the choice of using CT-Th as a screening tool [2]. But unlike China, CT-Th is not considered in the diagnostic criteria by most countries especially due to financial as well as operative issues.

\section{Summary}

Though viral pneumonia especially that caused by SARS-CoV-2 has typical or characteristic patterns on CT-Th yet significant overlapping features limit its routine use as a screening tool for diagnosing etiology of viral pneumonia including that of Covid-19. However, recognition of CT-Th features that may be characteristic or more commonly seen with one virus family than other may aid not only in initiating early empirical antiviral therapy but may also obviate injudicious use of antibiotic therapy.

\section{Conflict of Interest: NIL}

\section{References}

[1] Huang C, Wang Y, Li X, et al. (2020). Clinical features of patients infected with 2019 novel coronavirus in Wuhan, China. 
Lancet 2020, 395: 497-506.

[2] Lee EYP, Ng MY, Khong PL. (2020). Covid-19 pneumonia: what has CT taught us. Lancet Infect Dis. 2020, 20(4): $384-385$.

[3] Shi H, Han X, Jiang N, Cao Y, Alwalid O, Gu J, et al. (2020). Radiological findings from 81 patients with COVID-19 pneumonia in Wuhan, China: a descriptive study. Lancet Infect Dis 2020, 20(4): 425-434.

[4] Chung M, Bernheim A, Mei X, Zhang N, Huang M, Zeng X, et al. (2020). CT imaging features of 2019 novel coronavirus (2019-nCoV). Radiology 2020: 295(1): 202-207.

[5] Song F, Shi N, Shan F, Zhang Z, Shen J, Lu H, et al. (2020). Emerging coronavirus 2019-nCoV pneumonia. Radiology 2020, 295(1): 210-217.

[6] Ng M, Lee EYP, Yang J, Yang F, Li X, Wang H, et al. (2020). Imaging profile of the COVID-19 infection: radiologic findings and literature review. Radiol Cardiothorac Imaging 2020, 2(1): e200034.

[7] Bai HX, Hsieh B, Xiong Z, Halsey K, Choi JW, Tran TML, et al. (2020). Performance of radiologists in differentiating COVID-19 from viral pneumonia on chest CT. Radiology 2020: 200823. [Epub ahead of print]

[8] Pan F, Ye T, Sun P, Gui S, Liang B, Li L, et al. (2020). Time course of lung changes on chest CT during recovery from 2019 novel coronavirus (COVID-19) pneumonia. Radiology 2020: 200370. [Epub ahead of print].

[9] Koo HJ, Lim S, Choe J, Choi SH, SungH, Do KH. (2018). Radiographic and CT Features of Viral Pneumonia. RadioGraphics 2018, 38: 719-739.

[10] Ooi GC, Khong PL, Muller NL, You WC, Zhou LJ, Ho JC, et al. (2004). Severe acute respiratory syndrome: temporal lung changes at thin-section CT in 30 patients. Radiology 2004, 230: 836-44.

[11] Das KM, Lee EY, Al Jawder SE, Enani MA, Singh R, Skakni L, et al. (2015). Acute Middle East respiratory syndrome coronavirus: temporal lung changes observed on the chest radiographs of 55 patients. AJR Am J Roentgenol 2015, 205(3): W267-W27.

[12] Fang Y, Zhang H, Xie J, Lin M, Ying L, Pang P, et al. (2020). Sensitivity of Chest CT for COVID-19: Comparison to RT-PCR. Radiology 2020. https://doi.org/10.1148/radiol.2020200432. 